\title{
Delamination-Resistant Imperceptible Bioelectrode for Robust Electrophysiological Signals Monitoring
}

Wenjie Tang, ${ }^{a, \dagger}$ Yuxuan Zhou, ${ }^{a, \dagger}$ Shisheng Chen, ${ }^{a, \dagger}$ Shancheng Yu, ${ }^{a, \dagger}$ Yizhuo Yang, ${ }^{a}$ Jin Lin, ${ }^{a}$ Simeng Yin, ${ }^{a}$ Ying Ma, ${ }^{a}$ Benhui $H u^{a, b, *}$

a School of Biomedical Engineering and Informatics, Nanjing Medical University, Nanjing 211166, P. R. China. E-mail: hubenhui@njmu.edu.cn

b Jiangsu Key Laboratory of Xenotransplantation, Nanjing Medical University, Nanjing, 211166, P. R. China.

\section{Experimental section}

\section{Reagents}

PEDOT:PSS (high conductivity, 1.1-1.3 wt\%) and glycerol (suitable for cell culture) were purchased from Sigma-Aldrich. Polysorbate (Tween 80, suitable for cell culture) was obtained from Aladdin biochemical technology Co., Ltd. Poly(dimethylsiloxane) (PDMS) monomer and cure agent were supplied by Dow chemical company. The commercial $\mathrm{Ag} / \mathrm{AgCl}$ electrodes were purchased from Shanghai Kuakang Biotechnology Co., Ltd. 


\section{Preparation of DrIE}

In a typical experiment, glycerol of $100 \mathrm{wt} \%$ and polysorbate of $50 \mathrm{wt} \%$ were added into the PEDOT:PSS aqueous dispersion and vortexed at room temperature for $20 \mathrm{~min}$. Thin films $(\sim 300 \mathrm{~nm})$ were prepared by spin-coating the mixture on PDMS substrates at $600 \mathrm{rpm}$ for $60 \mathrm{~s}$, followed by drying at room temperature for $4 \mathrm{~h}$. The freestanding films $(\sim 20 \mu \mathrm{m})$ were obtained by dropping $70 \mu \mathrm{L}$ mixture into the PDMS moulds $(20 \times 5 \times 0.5 \mathrm{~mm})$ and drying overnight at room temperature. DrIE film $(\sim 2 \mu \mathrm{m})$ was obtained via dropping $20 \mu \mathrm{L}$ mixture into the PDMS moulds $(10 \times 10 \times 0.5 \mathrm{~mm})$ and drying at room temperature for $4 \mathrm{~h}$. The PDMS substrates and moulds were treated with oxygen plasma at $80 \mathrm{~W}$ for $30 \mathrm{~s}$ before spin-coating or dropping. The PDMS substrates were fabricated by spin-coating the mixture of PDMS monomer and cure agent at the weight ratio of $10: 1$ on the plastic plates at $1000 \mathrm{rpm}$ for $30 \mathrm{~s}$ and curing under $60^{\circ} \mathrm{C}$ for $2 \mathrm{~h}$.

\section{Measurements of chemical and morphological characteristics}

The Raman spectra were recorded on Horiba HR Evolution. The X-ray diffractograms (XRD) were performed on Bruker D8 Advance with wide angle $\left(5-80^{\circ}\right)$. UV-vis-NIR spectra were carried out on a Shimadzu UV 3600 plus in the wavelength range of 200-1200 nm. AFM images were collected by Bruker Dimension ICON with tapping mode.

\section{Measurements of electrical and mechanical properties}

The conductivities of the thin films supported on PDMS substrates with different components were measured through the four-point probe method with Keithley 
2450 source meter. The width and length of the thin films were obtained using a vernier caliper. The thickness of DrIE film was calculated according to the height difference of the height-distance curves measured by AFM. The electrical stability was measured using Keithley 2450 source meter and Criterion Electromechanical Test Systems (C42.503, MTS) at a tensile speed of 10 $\mathrm{mm} / \mathrm{min}$. The interfacial impedance was collected using LCR meter (IM 3533$01, \mathrm{HIOKI})$ in the range of $20 \mathrm{~Hz}-200 \mathrm{kHz}$, where two electrodes $(10 \times 10 \mathrm{~mm})$ were attached to the forearm with a distance of $2 \mathrm{~cm}$. The Young's modulus and elongation at break of electrodes were studied on freestanding films (thickness $\sim 20 \mu \mathrm{m}$, measured using an optical microscope) with Criterion Electromechanical Test Systems at a tensile speed of $10 \mathrm{~mm} / \mathrm{min}$. The stressstrain properties of our DrIE and commercial $\mathrm{Ag} / \mathrm{AgCl}$ attached PDMS substrates were characterized using the Criterion Electromechanical Test Systems.

\section{Measurements of adhesion force}

The adhesion force was measured through standard 90-degree peeling approach using Criterion Electromechanical Test Systems. A rectangle DrIE film of $10 \times 30$ $\mathrm{mm}$ was attached to clean porcine skin, and then perpendicularly detached from porcine skin at a speed of $6 \mathrm{~mm} / \mathrm{min}$. The adhesion force was calculated using maximum stable force and the DrIE film width. The peeling off process and skin irritation of our DrIE and commercial $\mathrm{Ag} / \mathrm{AgCl}$ electrode were observed after wearing for $12 \mathrm{~h}$. 


\section{Measurements of gas permeability}

$45 \mathrm{~mL}$ water was added in three centrifuge tubes which were covered with parafilm, our DrIE and nothing, respectively. The water volumes after $72 \mathrm{~h}$ were measured to evaluate gas permeability. The thermal mapping images of human forearm attached with our DrIE for 10 minutes were collected using thermal infrared imager (FLIRONE PRO).

\section{Monitoring of EMG signals}

The surface EMG signals were acquired using PowerLab (PL3516, AD Instruments) by placing three electrodes on the wrist flexors of right forearm. The DrIE was connected with test instruments by conductive silver adhesives and conductive tapes. In detail, the conductive silver adhesives were used to bond DrIE and conductive tapes, and the conductive tapes act as wires to connect with test instruments. The obtained raw EMG signals were band-passed $(10-500 \mathrm{~Hz})$ and amplified 1000 times by Differential AC Amplifier (1700, A-M System). The wrist flexion was measured using torque transducer during the monitoring process. Simultaneously, the EMG signals were recorded at 0 h, 2 h, 4 h, 8 h, and 12 h. The signal-to-noise ratio (SNR) was calculated by Matlab (MathWorks) through the equation intensity and background noise level. The EMG Hudgins' features during single-finger movements were analyzed by Matlab, including mean absolute value (MAV), number of slope sign (NSS), number of zerocrossing (NZC) and waveform length (WL). The movements of different fingers were classified by a cubic support vector machines (SVM) classifier. 


\section{Detection of EEG signals}

The EEG signals recording was performed on a healthy volunteer. Two DrIEs of $10 \times 10 \mathrm{~mm}$ were placed on the left forehead (FP1) and occipital (O1) positions served as cathode and anode, respectively. Another DrIE was attached to the mastoid bone behind the left ear as reference. The collected EEG signals were band-passed $(0.1-500 \mathrm{~Hz})$ and amplified 1000 times at a sampling rate of $10 \mathrm{kHz}$ using PowerLab. The raw data were analyzed using Matlab.

\section{Synchronous recording of SKNA and ECG signals}

The SKNA signals were acquired by placing two DrIEs of $10 \times 10 \mathrm{~mm}$ on the subclavicular area and a reference electrode on the right abdomen. The signals were processed with a bandpass filter $(300-1000 \mathrm{~Hz})$ and were recorded by PowerLab. The integrated SKNA (iSKNA) was obtained using LabChart software to express the signal intensity of SKNA. Simultaneously, the ECG signals were collected using a standard three-electrode system (RA, LA, LL). The collected signals were band-passed (10-1000 Hz) and amplified 1000 times at a sample rate of $10 \mathrm{kHz}$. The obtained ECG data were analyzed using LabChart to calculate heart ratio (HR).

\section{Recording of EOG singnals and real-time control of aircraft}

The EOG signals were collected by placing electrodes on the periocular area. One DrIE was placed on the forehead as reference electrode. Two DrIEs were laminated on up and down of the left eyeball as channel one to monitor vertical movements of the eyeball, and the other two DrIEs were placed on near the outer 
corner of the eyes as channel two to detect lateral motions of eyeball. The recorded signals were band-passed $(10-500 \mathrm{~Hz})$ and amplified 1000 times. The processed signals were classified into four commands through the amplitude using a threshold detector. The commands were input to the computer to control the movement of the aircraft in real time.

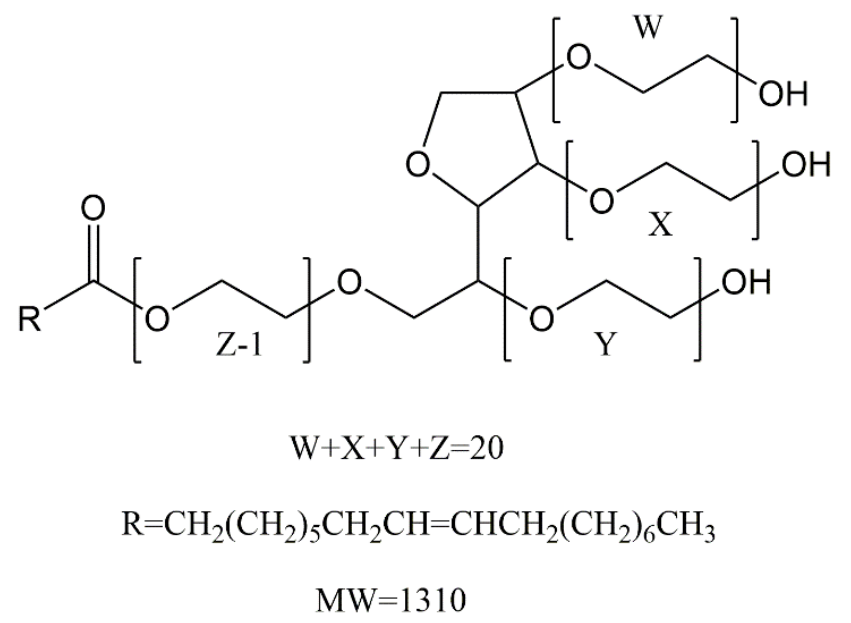

Figure S1. The chemical molecular structure of polysorbate (tween 80). 

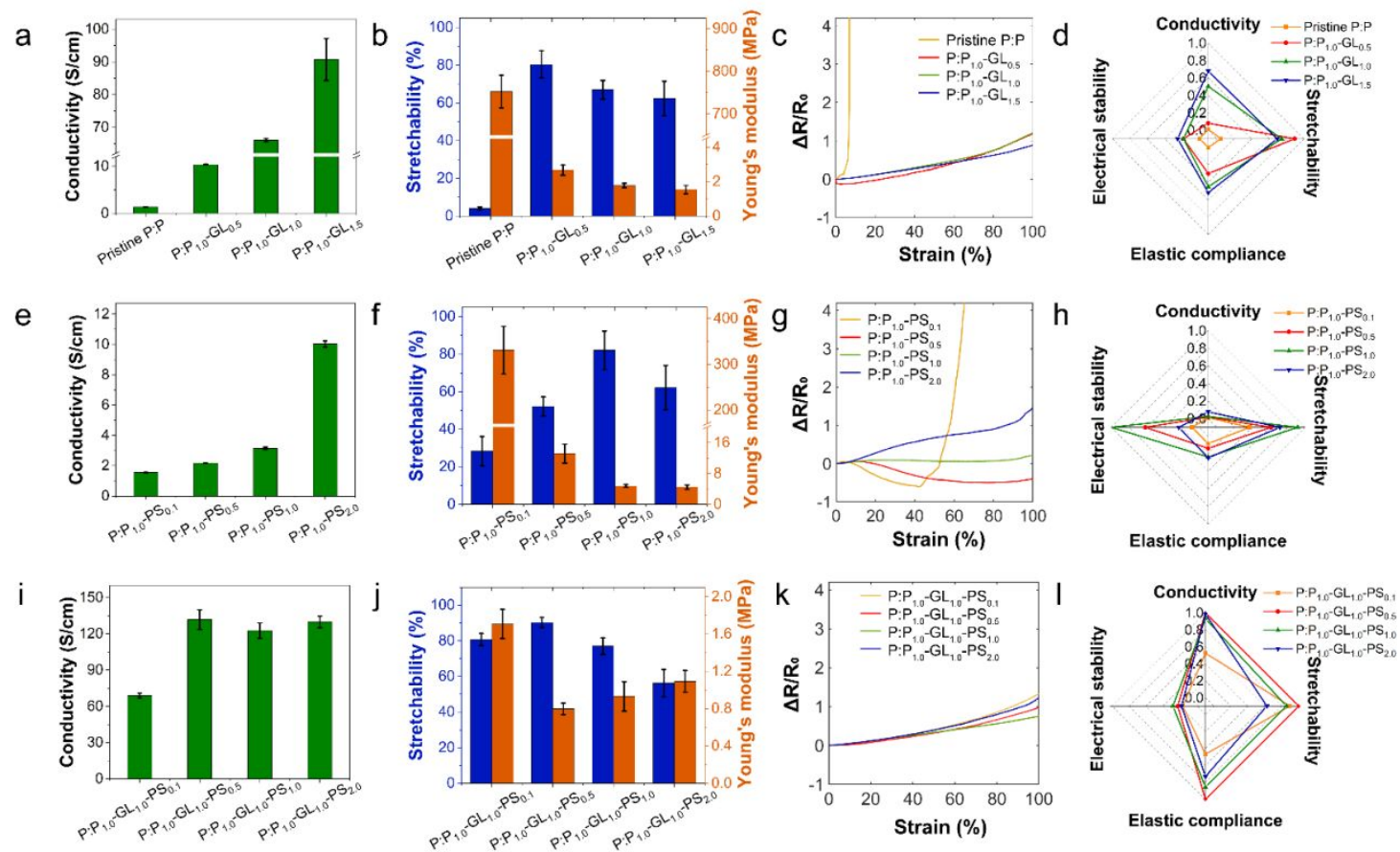

Figure S2. Optimization of the electrical and mechanical properties. (a, b) Conductivity, stretchability (elongation at break) and Young's modulus (elastic compliance) of PEDOT:PSS films with different glycerol contents. (c) Resistance change rate $\left(\Delta R / R_{0}\right)$ of glycerol doped PEDOT:PSS films supported on PDMS substrates during tensile strain. (d) Radar chart of four normalized factors. Similarly, (e-h) and (i-1) four factors and radar chart of PEDOT:PSS film treated with polysorbate or glycerol/polysorbate, respectively. Error bars show standard deviation, sample size $n=7$.

The synergistic effects of glycerol and polysorbate are quantitively studied based on four key factors affecting electrode performance, including conductivity, stretchability (elongation at break), elastic compliance (1/Young's modulus) and electrical stability (1/resistance change rate), whereby the weight ratio (wt\%) of PEDOT:PSS, glycerol and polysorbate is optimized following a constraint criterion as:

$$
\begin{gathered}
\max A(w t \%) \\
\text { s.t. Stretchability } \geq 60 \%
\end{gathered}
$$

where $A$ is the envelope area within concatenating curves on the radar chart with the axes of four key factors. The safe stretchability above $60 \%$ is set as the constraint condition because the insufficient stretchability of DrIE than human skin $(\sim 50 \%)$ may induce delamination or rupture of DrIE. ${ }^{1}$ The results show that enlarging the content of 
glycerol (1:1 weight ratio of glycerol : PEDOT:PSS) will lead to conductivity increasement from 1.34 S/cm to $66.07 \mathrm{~S} / \mathrm{cm}$ ( $\sim 2$ orders of magnitude) (Figure S2a). It is noteworthy that excessive amount of glycerol decreases the stretchability of doped PEDOT:PSS film to the unsafety zone (below 60\%) (Figure S2b). Therefore, the weight ratio of glycerol : PEDOT:PSS is chosen to be 1:1 to maximize the envelope area on the radar chart with the stretchability above $60 \%$ (Figure S2d). Moreover, with the increase of the polysorbate, the conductivity enhances from $1.34 \mathrm{~S} / \mathrm{cm}$ to $10.02 \mathrm{~S} / \mathrm{cm}$ while the Young's modulus decreases from $752.14 \mathrm{MPa}$ to $4.77 \mathrm{MPa}$. However, the stretchability is enhanced to maximum value ( $80 \%)$ only when the ratio of PEDOT:PSS : polysorbate is 1:1 (Figure S2f). Thus, adding the glycerol or polysorbate separately can only improve either the electrical or mechanical performance of DrIE, resulting in a compromise in optimizing both the conductivity and stretchability. Interestingly, the addition of the glycerol and polysorbate can synergistically enhance the conductivity $(131.78 \mathrm{~S} / \mathrm{cm})$ and stretchability $(\sim 90 \%)$ of our DrIE when the ratio of PEDOT:PSS : glycerol : polysorbate is 1:1:0.5 (Figure S2i,j). The decrease of Young's modulus ( 0.8 $\mathrm{MPa}$ ) of our DrIE contributes to the conformable contact with the skin. Last but not least, DrIE films treated with proper amount of glycerol (weight ratio of PEDOT:PSS to glycerol: 1:0.5 1:1.5) and polysorbate (weight ratio of PEDOT:PSS to polysorbate: 1:0.5 1:2) show a lower resistance change rate during tensile stretching than the pristine film, exhibiting higher electrical stability against strain to satisfy actual application requirements (Figure S2c,g,k). Taken together, the optimum weight ratio of PEDOT:PSS : glycerol : polysorbate is 1:1:0.5 according to the maximum envelope 
area of radar plot (Figure S2d,h,1).
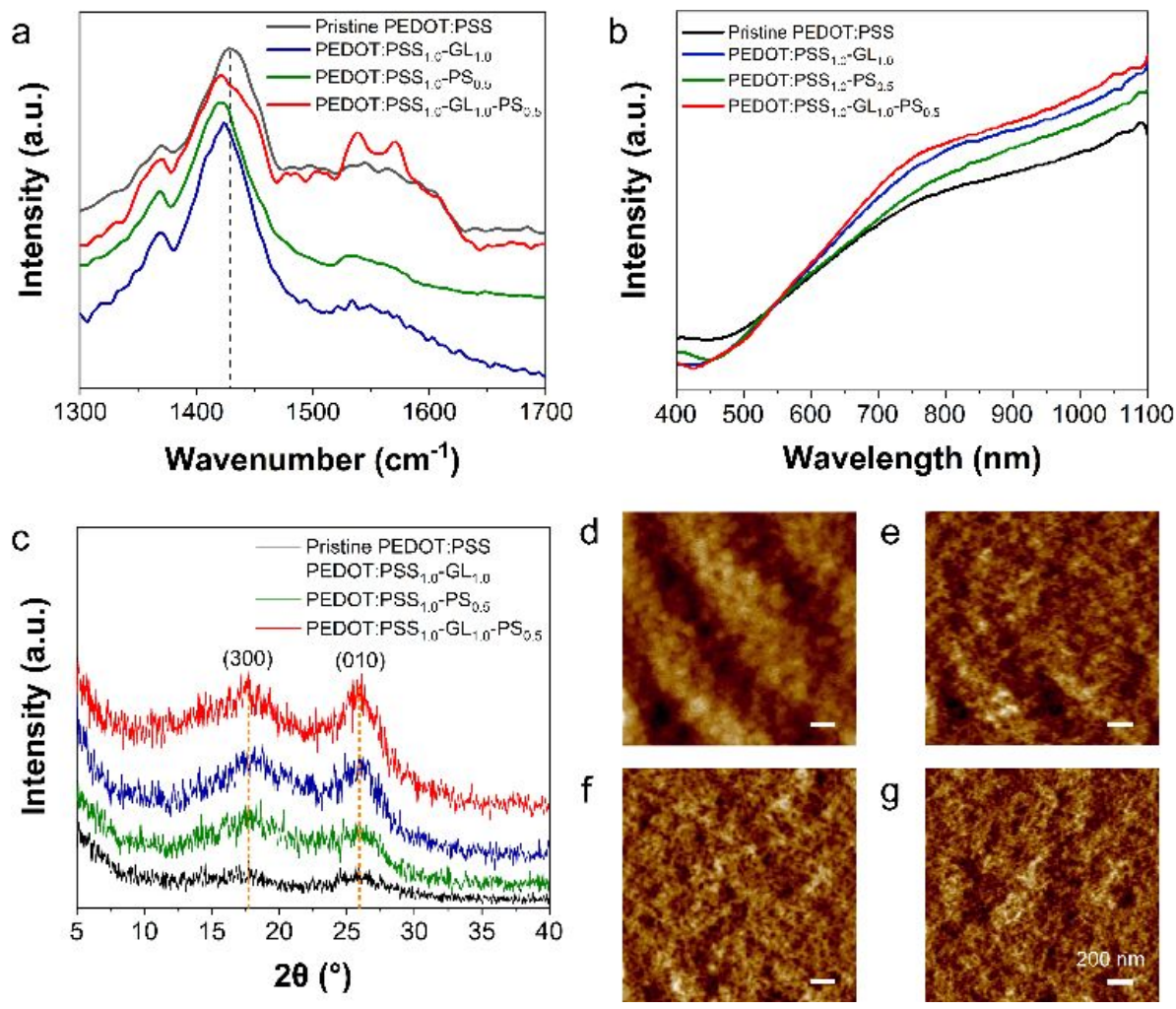

Figure S3. Chemical and morphological characteristics of PEDOT:PSS films. (a-c) The Raman spectra (a), UV-Vis-NIR spectra (b) and XRD spectra (c) of different PEDOT:PSS films doped with glycerol and polysorbate. (d-g) The AFM images of pristine PEDOT:PSS film (d), PEDOT:PSS films doped with glycerol (e), polysorbate (f) and glycerol/polysorbate (g). All scale bars, $200 \mathrm{~nm}$.

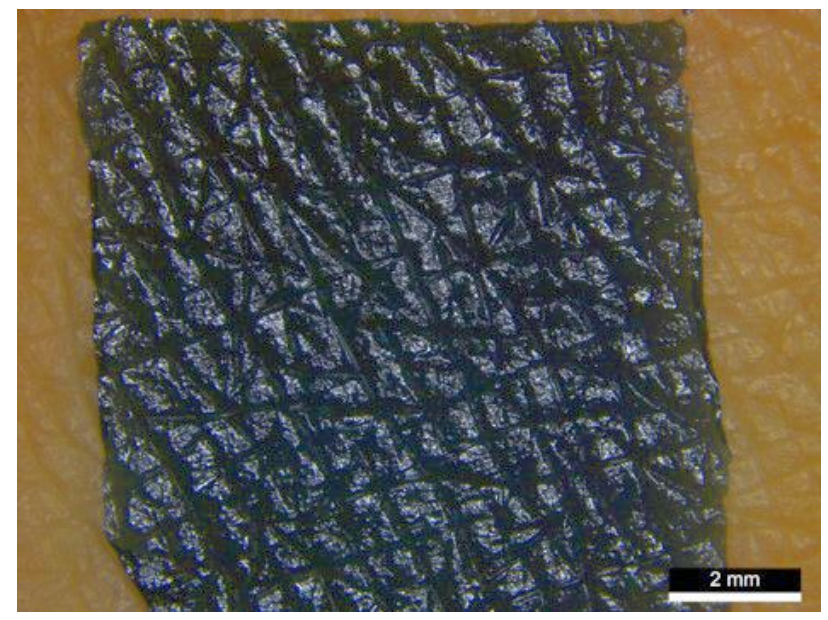

Figure S4. The optical microscope image of delamination-resistant imperceptible bioelectrode (DrIE) attaching on human forearm skin. The scale bar, $2 \mathrm{~mm}$. 
a
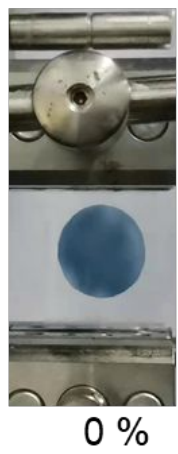

b

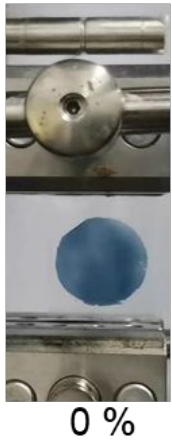

C

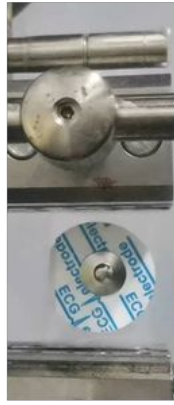

$0 \%$

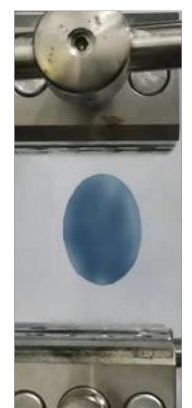

$30 \%$
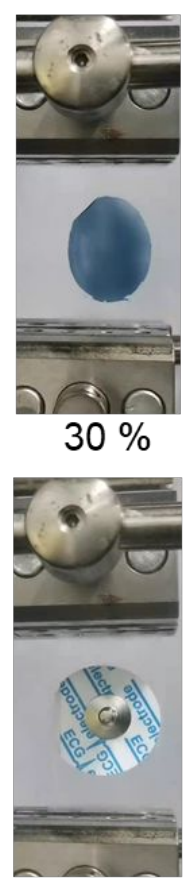

$30 \%$

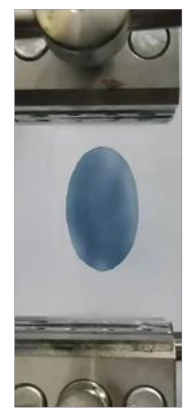

$50 \%$

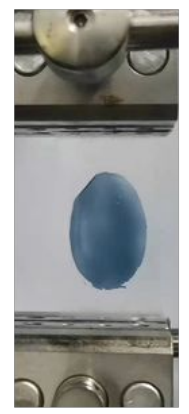

$50 \%$

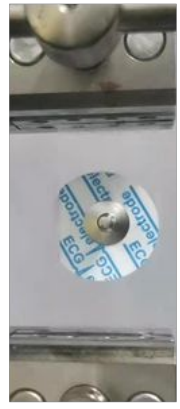

$50 \%$
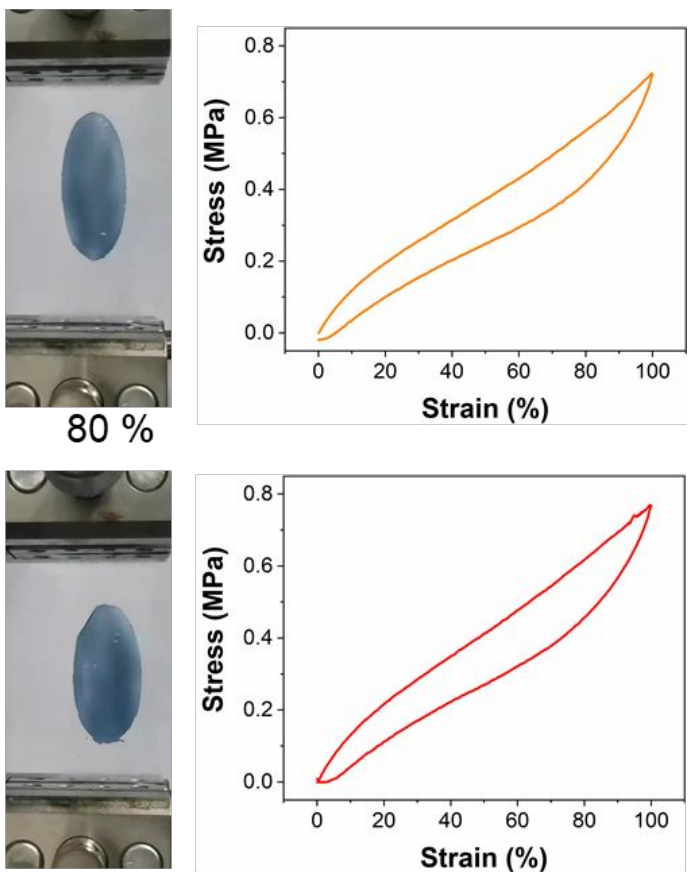

$80 \%$

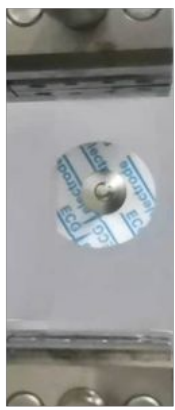

$80 \%$

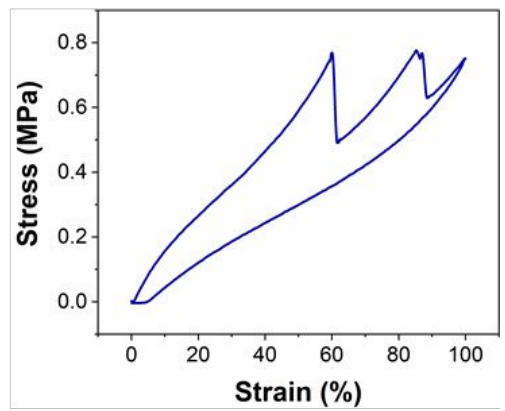

Figure S5. Photographs for various strains of the different electrodes attaching on skin substitute (PDMS-based substrate) and stress-strain curves. (a) Before contaminated DrIE. (b) After contaminated DrIE. (c) Commercial Ag/AgCl electrode.
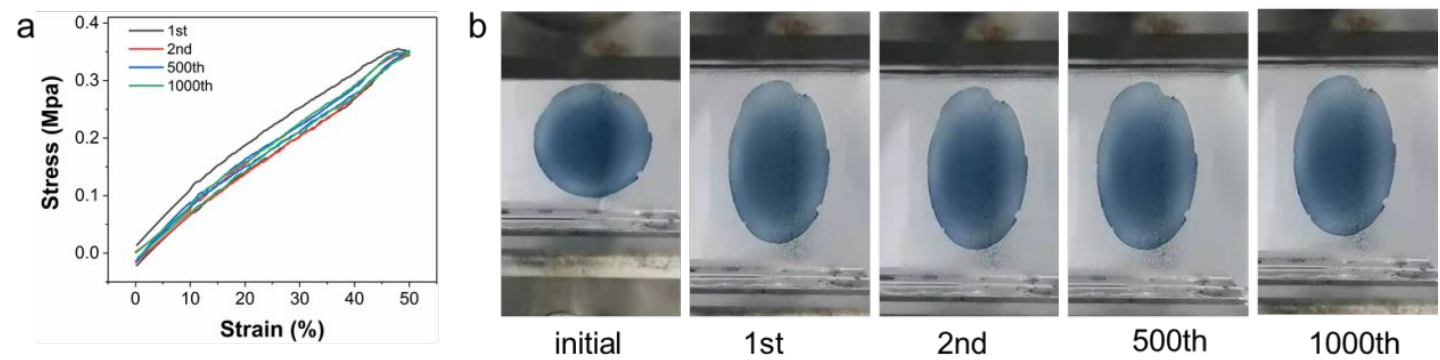

Figure S6. The stress-strain curves of skin substitute (PDMS film, thickness $100 \mu \mathrm{m}$ ) attached with DrIE during 1000 cycles (a) and corresponding photography (b). The tensile speed was $50 \mathrm{~mm} / \mathrm{min}$. 
a

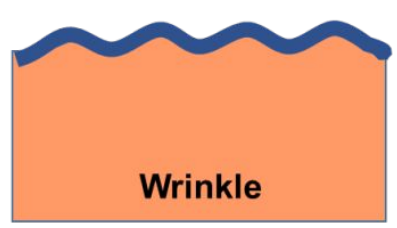

Wrinkle b

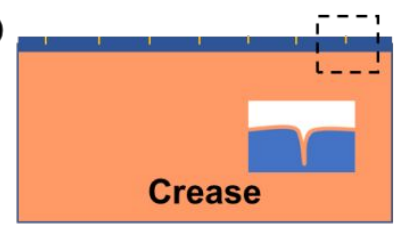

Crease
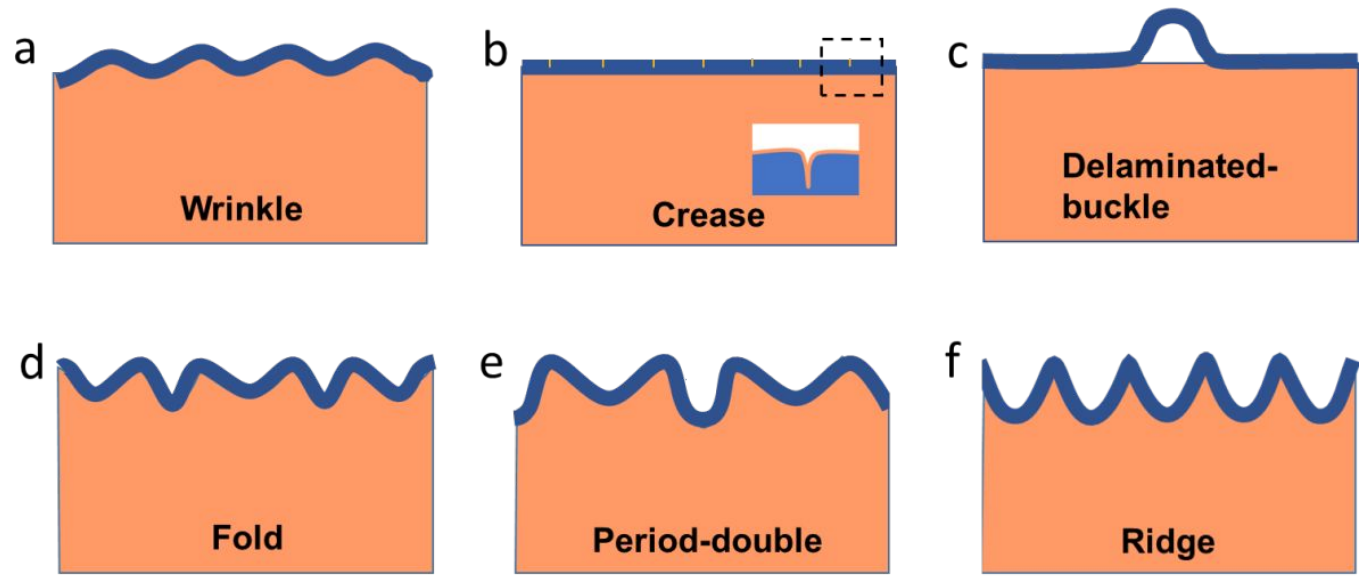

Figure S7. Schematics of growth-induced interfaces: (a) Wrinkle; (b) Crease; (c) Delaminatedbuckle; (d) Fold; (e) Period-double; (f) Ridge. ${ }^{2}$

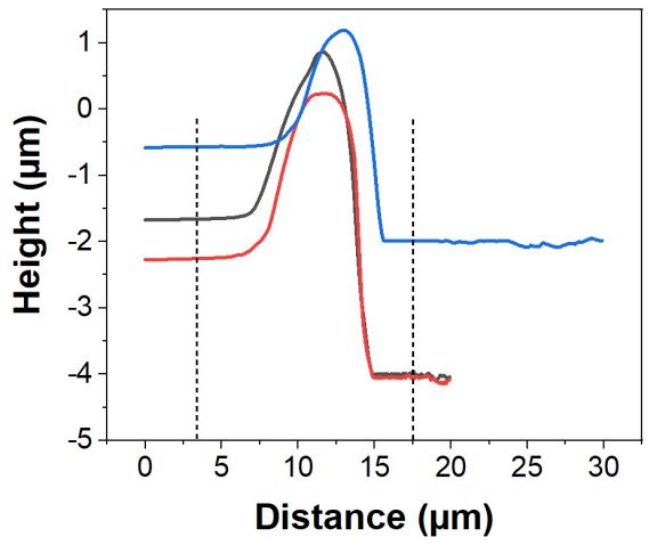

Figure S8. The thickness of DrIE is measured using AFM with tapping mode. The average thickness, $1.85 \mathrm{um}$

a
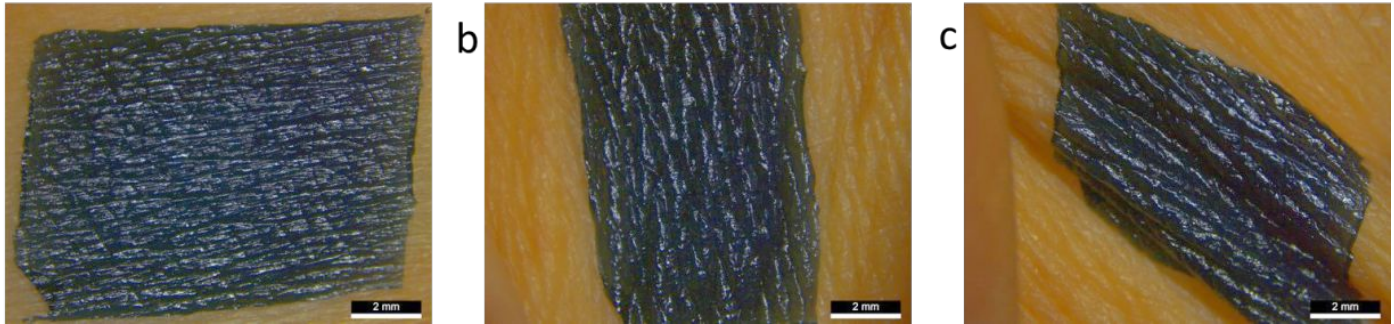

Figure S9. The optical images of DrIE under various skin deformation, including stretching (a), compressing (b), and twisting (c). The scale bar, $2 \mathrm{~mm}$. 

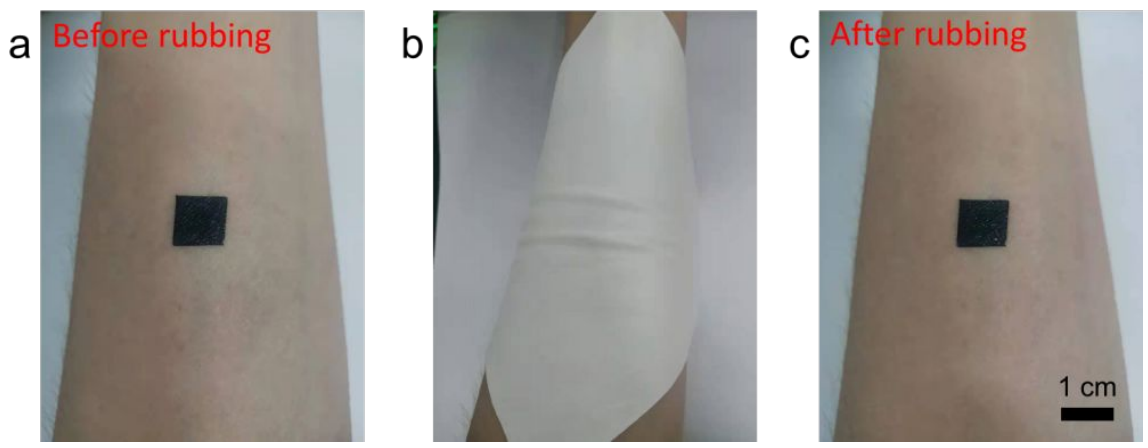

Figure S10. The resilience against clothes friction. (a) The photograph of the DrIE attached to forearm skin. (b) Rubbing the attached DrIE for 100 times with non-woven fabric. (c) The photograph of the DrIE after rubbing. The scale bar, $1 \mathrm{~cm}$.

a

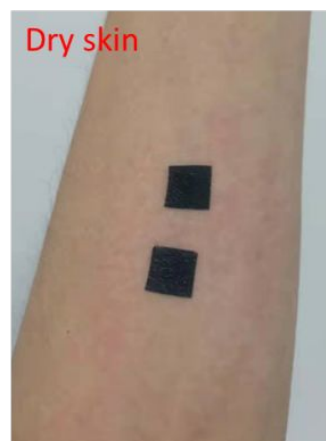

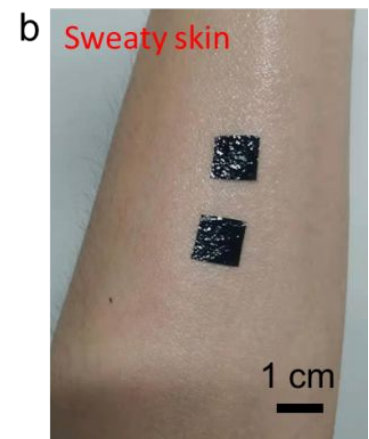

Figure S11. The photograph of DrIEs attached to dry forearm skin (a) and sweaty skin (b). The scale bar, $1 \mathrm{~cm}$.

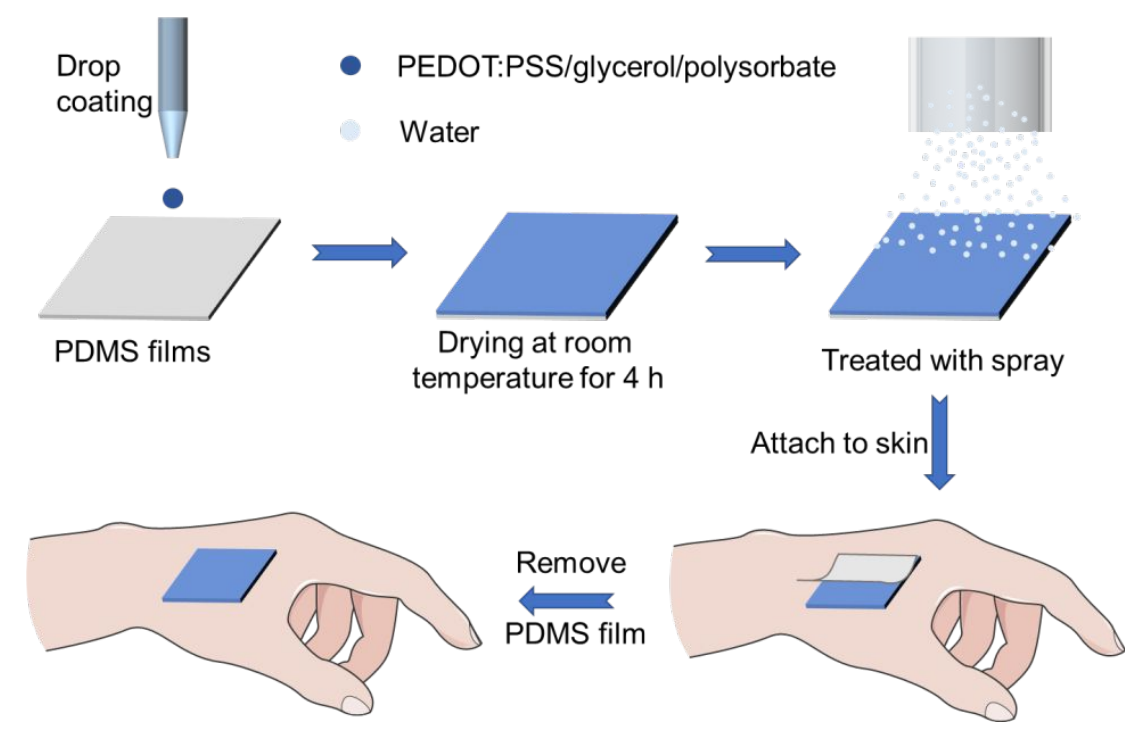

Figure S12. The fabrication and attachment process of the DrIE film: Firstly, drop-coating the mixture of PEDOT:PSS, glyceryl and polysorbate on a PDMS substrate; Secondly, drying at room temperature for $4 \mathrm{~h}$; Thirdly, treating DrIE with water spray; Fourthly, attaching to the skin; Finally, removing the PDMS substrate. 

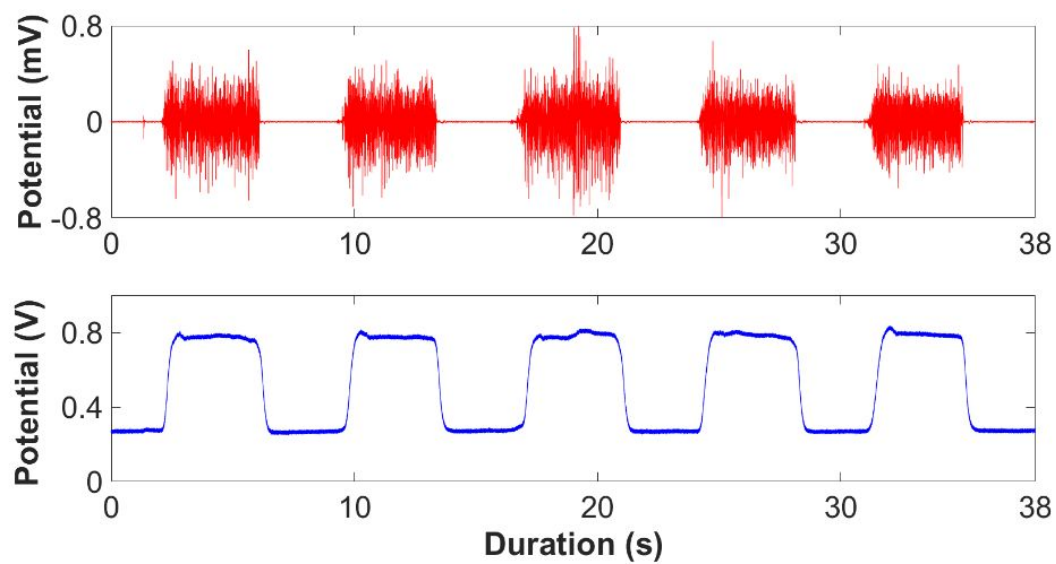

Figure S13. Recording of EMG signals using commercial $\mathrm{Ag} / \mathrm{AgCl}$ electrode. The $\mathrm{SNR}$ is 34.72 $\pm 0.68 \mathrm{~dB}$.
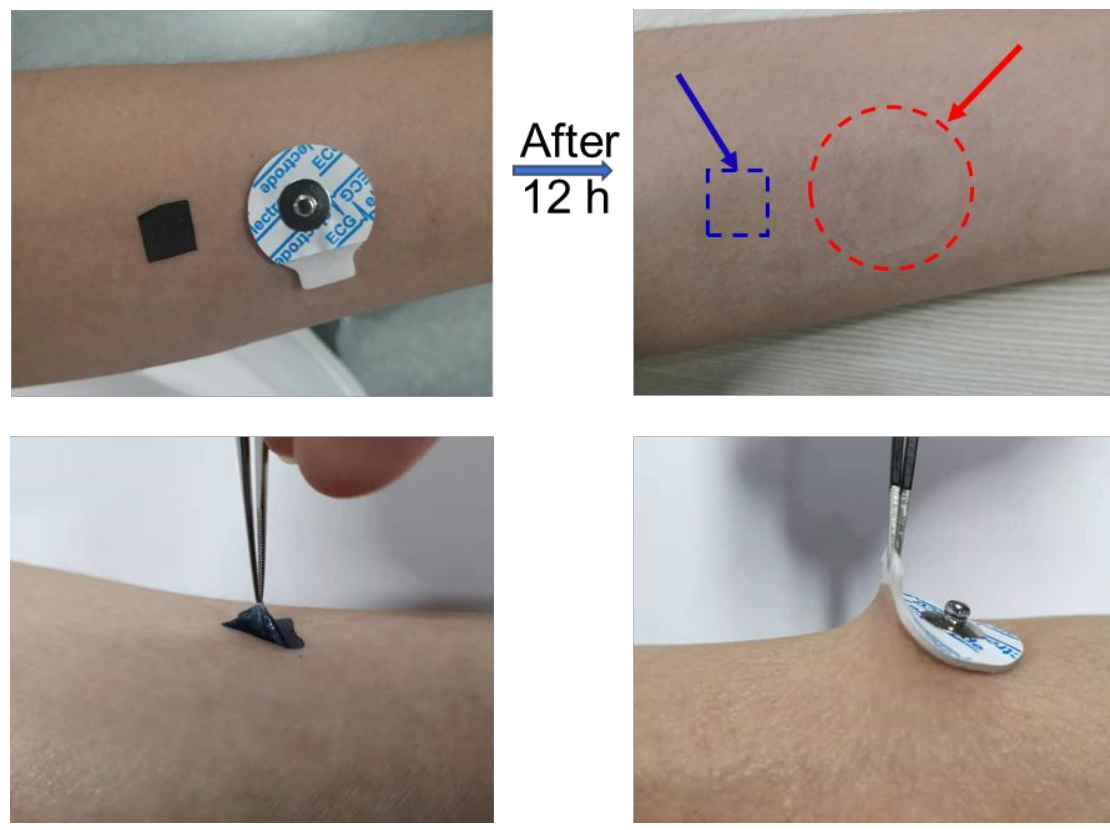

Figure S14. Photographs of the DrIE and commercial $\mathrm{Ag} / \mathrm{AgCl}$ electrode attached skin before and after peeling off. Our DrIE can be easily peeled off without dermatological irritation compared with $\mathrm{Ag} / \mathrm{AgCl}$ electrode. 
a

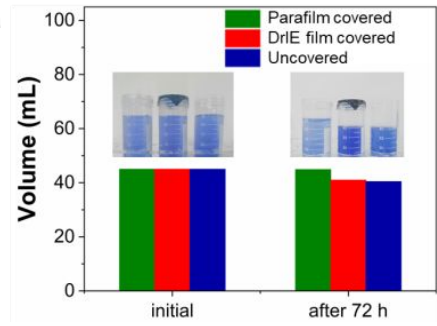

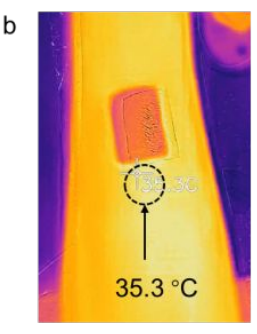
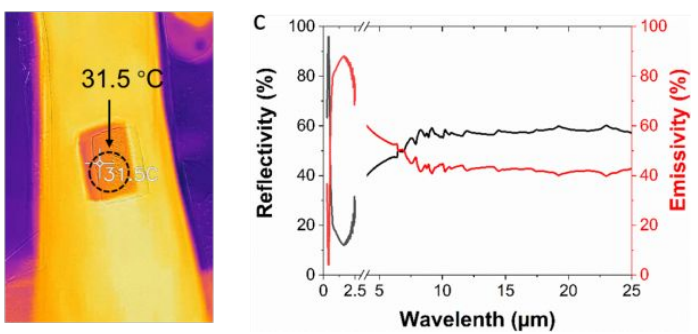

Figure S15. The experiment of gas permeability. (a) Comparison of the water volume in the parafilm covered, our DrIE covered, and uncovered centrifuge tubes before and after $72 \mathrm{~h}$ evaporation at room temperature, respectively. (b) Thermal mapping images of our DrIE attaching to human forearm. (c) The reflectivity and emissivity spectra of the DrIE.

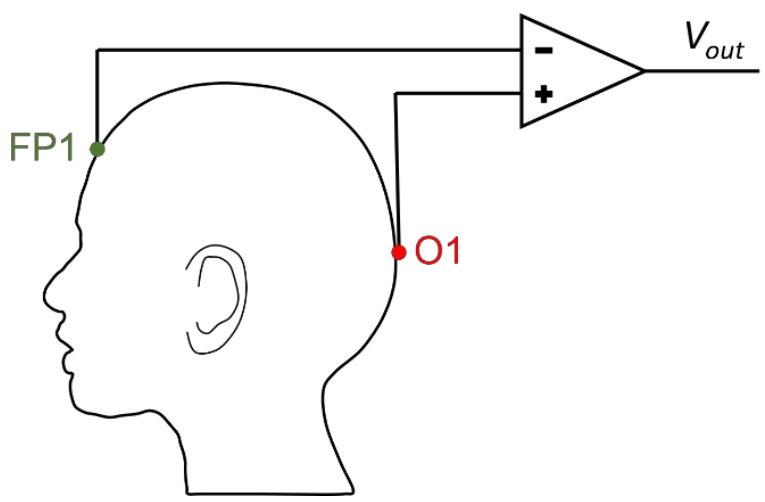

Figure S16. Wiring diagram of EEG signals recording.

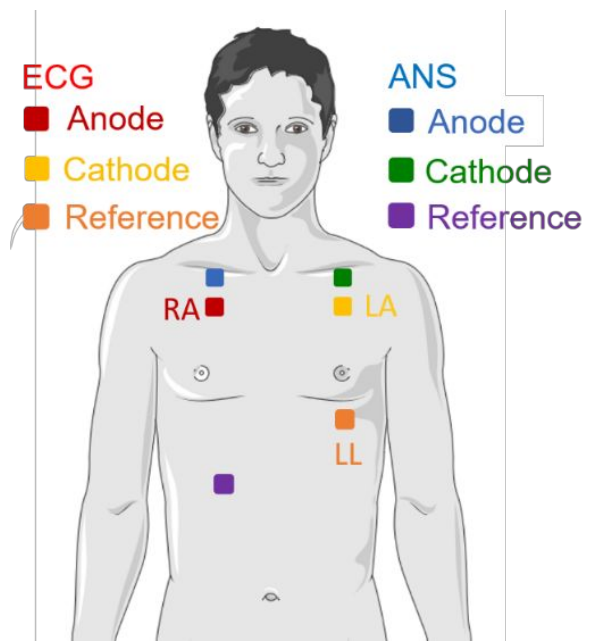

Figure S17. Schematic illustration for electrode attached positions of ECG and SKNA signals

recording. 


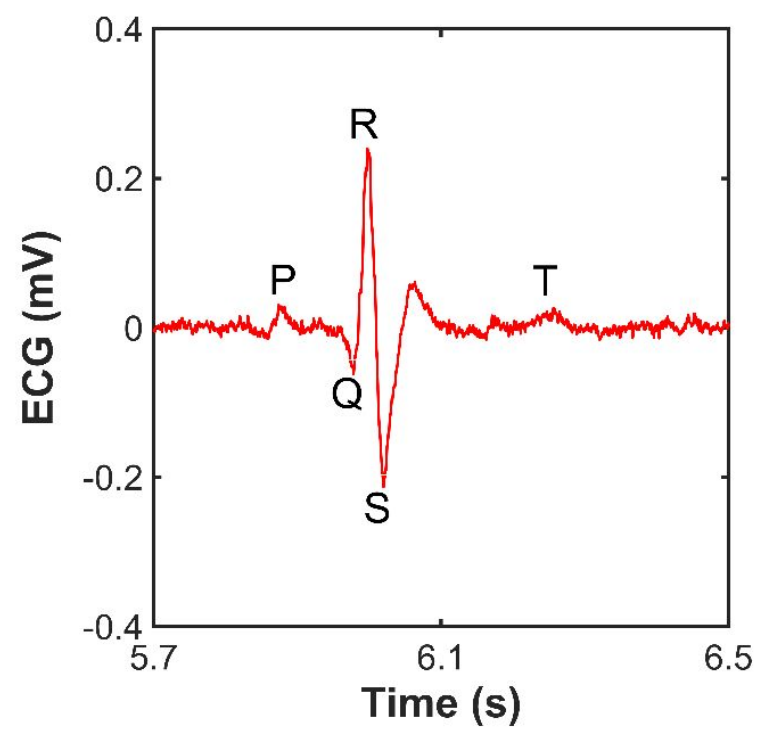

Figure S18. A single ECG waveform recorded by our DrIE.

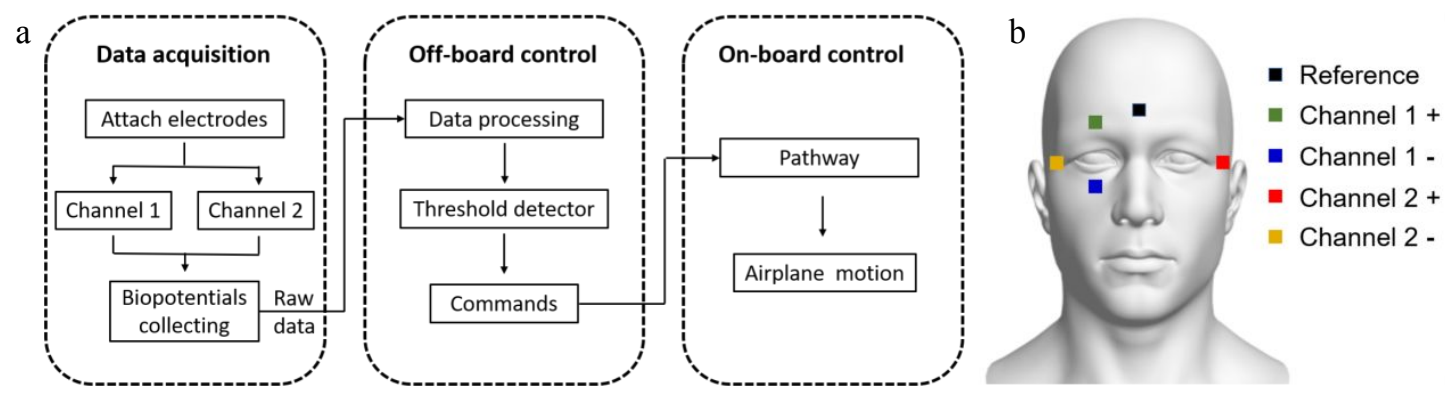

Figure S19. The flowchart of EOG signals acquisition, classification and interactive manipulation

(a) and schematic diagram of the electrode attached positions during EOG signals recording (b). 
Table S1. The SNR and adhesion force of the different electrodes.

\begin{tabular}{|c|c|c|c|c|c|}
\hline Electrode & Electrode type & $\begin{array}{c}\text { Adhesion force } \\
(\mathrm{N} / \mathrm{cm})\end{array}$ & SNR (dB) & Reference \\
\hline 1 & AgNW/SEBS & dry & 0.4 & 20.7 & 3 \\
\hline 2 & Graphene/PI & dry & 0.29 & 11.8 & 4 \\
\hline 3 & TI/AU/PI & dry & 0.0078 & 10.93 & 5 \\
\hline 4 & Au/parylene & dry & 0.135 & 21.09 & 6 \\
\hline 5 & RGO/PDMS & dry & 0.0713 & 16.8 & 7 \\
\hline 6 & Au/PI & dry & 0.0025 & 23 & 8 \\
\hline 7 & PMMA/PI/Au & dry & 0.47 & 13.2 & 9 \\
\hline 8 & Alg-PAAm & wet & 0.9 & 25 & 10 \\
\hline 9 & AgLMP & wet & 0.1 & 10 & 11 \\
\hline 10 & PSGO-PEDOT & wet & 0.2 & 5 & 12 \\
\hline 11 & CoupOn & wet & 0.15 & 32.2 & 13 \\
\hline 12 & Au/Silk & wet & 0.8 & 17.17 & 14 \\
\hline 13 & DrIE & dry & 0.013 & 35.23 & This work \\
\hline
\end{tabular}




\section{Supporting references}

1. Lu, B.; Yuk, H.; Lin, S.; Jian, N.; Qu, K.; Xu, J.; Zhao, X. Pure PEDOT:PSS Hydrogels. Nat. Commun. 2019, 10, 1043.

2. Wang, Q.; Zhao, X. A Three-Dimensional Phase Diagram of Growth-Induced Surface Instabilities. Sci. Rep. 2015, 5, 8887.

3. Xu, Y.; Sun, B.; Ling, Y.; Fei, Q.; Chen, Z.; Li, X.; Guo, P.; Jeon, N.; Goswami, S.; Liao, Y.; Ding, S.; Yu, Q.; Lin, J.; Huang, G.; Yan, Z. Multiscale Porous Elastomer Substrates for Multifunctional On-Skin Electronics with PassiveCooling Aapabilities. P. Natl. Acad. Sci. USA 2020, 117, 205-213.

4. Kwon, Y. T.; Kim, H.; Mahmood, M.; Kim, Y. S.; Demolder, C.; Yeo, W. H. Printed, Wireless, Soft Bioelectronics and Deep Learning Algorithm for Smart Human-Machine Interfaces. ACS Appl. Mater. Interfaces 2020, 12, 4939849406.

5. Kim, N.; Lim, T.; Song, K.; Yang, S.; Lee, J. Stretchable Multichannel Electromyography Sensor Array Covering Large Area for Controlling Home Electronics with Distinguishable Signals from Multiple Muscles. ACS Appl. Mater. Interfaces 2016, 8, 21070-21076.

6. Nawrocki, R. A.; Jin, H.; Lee, S.; Yokota, T.; Sekino, M.; Someya, T. SelfAdhesive and Ultra-Conformable, Sub-300 nm Dry Thin-Film Electrodes for Surface Monitoring of Biopotentials. Adv. Funct. Mater. 2018, 28, 1803279.

7. Li, Z.; Guo, W.; Huang, Y.; Zhu, K.; Yi, H.; Wu, H. On-Skin Graphene Electrodes for Large Area Electrophysiological Monitoring and Human- 
Machine Interfaces. Carbon 2020, 164, 164-170.

8. Jeong, J. W.; Yeo, W. H.; Akhtar, A.; Norton, J. J.; Kwack, Y. J.; Li, S.; Jung, S. Y.; Su, Y.; Lee, W.; Xia, J.; Cheng, H.; Huang, Y.; Choi, W. S.; Bretl, T.; Rogers, J. A. Materials and Optimized Designs for Human-Machine Interfaces via Epidermal Electronics. Adv. Mater. 2013, 25, 6839-6846.

9. Jang, K. I.; Jung, H. N.; Lee, J. W.; Xu, S.; Liu, Y. H.; Ma, Y.; Jeong, J. W.; Song, Y. M.; Kim, J.; Kim, B. H.; Banks, A.; Kwak, J. W.; Yang, Y.; Shi, D.; Wei, Z.; Feng, X.; Paik, U.; Huang, Y.; Ghaffari, R.; Rogers, J. A. Ferromagnetic, Folded Electrode Composite as a Soft Interface to the Skin for Long-Term Electrophysiological Recording. Adv. Funct. Mater. 2016, 26, 7281-7290.

10. Pan, L.; Cai, P.; Mei, L.; Cheng, Y.; Zeng, Y.; Wang, M.; Wang, T.; Jiang, Y.; Ji, B.; Li, D.; Chen, X. A Compliant Ionic Adhesive Electrode with Ultralow Bioelectronic Impedance. Adv. Mater. 2020, 32, 2003723.

11. Stauffer, F.; Thielen, M.; Sauter, C.; Chardonnens, S.; Bachmann, S.; Tybrandt, K.; Peters, C.; Hierold, C.; Voros, J. Skin Conformal Polymer Electrodes for Clinical ECG and EEG Recordings. Adv. Healthc. Mater. 2018, 7, 1700994.

12. Gan, D.; Huang, Z.; Wang, X.; Jiang, L.; Wang, C.; Zhu, M.; Ren, F.; Fang, L.; Wang, K.; Xie, C.; Lu, X. Graphene Oxide-Templated Conductive and Redox-Active Nanosheets Incorporated Hydrogels for Adhesive Bioelectronics. Adv. Funct. Mater. 2020, 30, 1907678.

13. Cai, P.; Wan, C.; Pan, L.; Matsuhisa, N.; He, K.; Cui, Z.; Zhang, W.; Li, C.; 
Wang, J.; Yu, J.; Wang, M.; Jiang, Y.; Chen, G.; Chen, X. Locally Coupled Electromechanical Interfaces Based on Cytoadhesion-Inspired Hybrids to Identify Muscular Excitation-Contraction Signatures. Nat. Commun. 2020, 11, 2183.

14. Chen, G.; Matsuhisa, N.; Liu, Z.; Qi, D.; Cai, P.; Jiang, Y.; Wan, C.; Cui, Y.; Leow, W. R.; Liu, Z.; Gong, S.; Zhang, K. Q.; Cheng, Y.; Chen, X. Plasticizing Silk Protein for On-Skin Stretchable Electrodes. Adv. Mater. 2018, 30, 1800129. 